\title{
DOCUMENTOS INÉDITOS, RELATIVOS AO RIO DE JANEIRO (II).
}

\section{(Continuação) .}

A. H. U. - Rio de Janeiro - 1619 .

Señor.

Rui Vas pinto Capitão desta Cap.a por Vosa mag.d fiqa asistindo em seu cargo com muita satisfação. de todos asi nas materias que se oferecem de Guerra como no Procedimento do Governo Veo com sua molher e filhos fiqo muto pobre e pelo zello que tem do serviço de Vmg merese fazerlhe Vmg.de merse e nos de nosa parte pela pax e quietasão com que governa a todos. pedimos a Vng.de nos faça merse de acabamte seu tempo melhoralo pera que asi posa acodir ao Remedio de sete filhos e filhas com que fiqa que nos lhe não podemos pagar os serviços que a Vmg.de faz a esta Cap.a senão com a lembrança quea Vmg.de fazemos e pedimos de merce guarde Deos a catholica pesoa de Vmg.de Por largos anos, Rio de Jan.ro em Camara 26 de abril de 1619. Alvaro da Costa escrivão da Camara o escrevi.

Fr.co allz da fonceq.a

Estevão daraujo

Simão lobo

m.l botelho

fr.co de mariz

Ant.o pachequo Calhejros.

No verso: Rio de Jan.ro 26 de Abril de 619.

Da Camara da Cap.a do Rio de Jan.ro Sobre abonação de Ruj Vaz pinto q. servio alj de Capitã. 
A. H. U. - Rio de Janeiro - 1619 .

Sñor

halem de Por outras vias o ter já feito e avizado a? vmag.de do prosedimto de Rui vas pinto Capitáo mor desta sidade o torno agora a fazer dando conta a vmag.de do prezente e he que o dito Capitáo mor se intermete com tanta forsa na fazenda de vmag.de e com os ofisiais dela que não poso deixar sem embarguo de ter avizado ao g.dor geral deste estado e ao provedor. mor da fazenda de vmg.de dizo fazelo em espesial a vmag.de porque são. tantas as eistroções e desordeñs que o dito Capitão mor comete no tocante a este Particular que se se lhe não atalha com o Remedio serto não dixara de dar em hua: grande falta que alem de querer com qualquer Pequena. ocazião guastar o que lhe parese da fazenda de vmag.de querendo me obriguar com forças e imjustçia a dar lha como o fez em hua jornada que fes ao cabo frio na qual guastou mais de sento e sincoenta mil Reis.quer tão bem que as praças dos soldados q. vmag.de tem nas fortalezas desta sidade lhe paguem a ele não tendo nas ditas. fortalezas mais que treze soldados o anno pasado e este prezente dezoito ou dezanove e todas as mais Praças come mortas e fas delas o que quer e sobre eu lhe ir a iso. ha mão me sustendeu do carguo de Provedor que tenho Por vmag.de de sua fazenda anexando me e molestando. me em tudo q.to pode e me fes ir ha baya e a pernãobuquo Por Rimir minha anexação guastando m.to de mjnha. faeznda vindo porem Rimido e Restituido a meu carguo e aguora de ordinario com qualquer Pequena ocazião me manda loguo ameasar com suspensõis e que me ade embarquar a ese Rejno Remetido e vmag.de e não ao g.dor geral Por niso me meter mais pavor e medo cuidando lhe eide fazer a vontade e he tão desordenado nestes particulares que diz que ade fazer o que quizer e que ningué lho ade impedir que soo a vmag.de ade dar conta e lha ade tomar e não outrĕ e asi $\mathrm{m}$. tas vezes estão as fortalezas de vmag.de despovoadas de soldados e outras com dous ou tres som.te e sem condestabeis nem bombardejros tudo Por seu // consentim.to e aRisquado a hum mau Recado Por Rezão do que tem comiguo mil descontos Por Ihe dizer tenho ordem do g.dor geral e do Provedor mor da faenda de vmag.de como de efeito a tenho Para lhe não levar em conta os guastos do cabo frio da jornada que la fes e as praças mortas dos soldados que come c Por eu mandar notefiquar aos soldados asistisem nas ditas fortalezas pois comĕ o dr.o de vmag. de Porque os que não asistisem Ihes não avia de mandar paguar o dito 
Capitão mor me mandou Por Pena que avia de suspender me se mais entendia com ós seus soldados e aguora nesta folha $q$. ade sair fiquo com Reseo dos emcontros que com elle eide ter Porque não eide Paguar mais que aos que autualm.te servem comforme a ordem q. tenho do g.dor geral e Provedor mor vmag.de se sirva mandar acudir e atalhar a estes danos com o Remedio que lhe pareser comvem e com brevidade Porque me não deixa o dito Capitão mor eizersitar meu carguo nem servir a vmag.de como qomvem a não som.te nestas materias mas tãobem no tocante aos navios nos quais entende e com os mestres deles anexando os e molestando os e dilatando-lhes suas viageñs não lhes querendo dar as lisenças p.a as fortalezas e metendo lhes guardas de sua mão impedindo me guardar meu Refim. to de que tudo Rezulta Perda e dano ha fazenda e direitos de vmag.de Porque com este seu Prosedim.to fogem os homeñs de vir a este Porto como dantes fazião e desta verdade emvio a vmag.de sertidois com esta g.de des a catholiqua e Real Pesoa de vmag.de Rio de jan.ro 14 de maio de 1619 .

Dioguo lopes de bulhão.

No verso: Rio de Janr.o - 14 de mayo de 619 .

Carta do Provedor da faz.a em q. ayisa do procedim. to de Ruy vaz Pinto e como se intremete nas matt.as da faz.a.

$*$

A. H. U. - Rio de Janeiro - 1619 - Doc. anexo.

Auto que mandou fazer.o provedor da fazenda de sua mag.de Di.o Lopes de bulhão.

Anno do nasimento de noso snôr Jezu Cristo de mil e seissentos e desasete, anos aos treze dias do mes de dezembro da dita era nesta sidade de são Sebastião do Rio de jan.ro na alfandega dela forão os Reverendos padres da companhia de ju do Coleio desta sidade predir ao provedor da fazenda de Sua mag.de diogo lopes de bulhāo por hua petisão feita en sinco dias do mes asima que vaj junta en como erão chegados ao Cabo frio sem casais de indios que ven a fazer numero de coatrosentos he sincoenta pesoas pouco mais ou menos os coais vierão por ordem do snõr governador geral don luis de sousa e qomforme a provisão que tinhào do dito Snõr governador mandava a ele provedor dese tudo o que fose nesesario para provimento dos ditos indios pelo que lhe pedirão the mandase dar quinhentos Alqueires de farinha Por coanto os ditos indios estavão sen teren que comer. o dito provedor 
Respondeo aos ditos Reverendos padres que na terra não avia farinha nem quon que se pudese conprar dado que ouvese mas quen estava no porto desta sidade hun navio que viera da Cananea diguo da Capitania de São Visente com tres mil alqueires de farinha que avia de ir para angola com a mais carga que avia de tomar que lhos pediria emprestados ate meado Janejro $\mathrm{E}$ lhos tomaria a dar .E logo o dito provedor mandou chamar o mestre do dito navio e lhe pedio a farinha na forma sobredita ao que Respondeo o dito mestre que lha não podia dar por ser de partes mas que se lha pedisen por orden de petisão con papeis para sua descargua que a daria e sendo aos catorze dias do dito mes o Capitan mor Rui vas pinto veo a dita Alfandega aonde o dito provedor e almoxarife e Eu escrivão estavamos prezentes dise como hera informado que per ordem dele dito provedor se hia a hun navio tomar farinha pera serviso de sua mag.de he que asi mandava a ele dito provedor nảo tomase a tal farinha nem nenhum navio para a levaren ao Cabo frio aonde os ditos indios estão sen sua orden per coanto a jurisdisão do mar e da terra hera sua he que tanto hera asy que seo proprio governador geral viera a esta sidade e Capitania senão avia de meter en sua Jurisdisão he que se hele dito provedor mandava faser as ditas diligensias o avia de suspender e os mais // ofisiais que as fisesen o que visto pelo dito provedor mandava a min escrivão fisese heste auto en que ele dito provedor asinou con os mais oficiais. E Eu escrivão o fis he o escrevy, Rafael de Carvalho, dioguo lopes de bulhão o Almoxarife luis de mejreles barboza C - Dis o procurador do coleio da conpanhia de Jezu desta sidade do Rio de jan.ro que no Livro do almoxarifado Desta sidade esta Resistada hua provisão pela coal e snor governador geral manda a Vosa merse de todo o nesesario para ha situasão e conservasáo da aldeia que ora novamente se setion no cabo frio $E$ per que os novos moradores são chegados de novo e não ten ainda Rosarias proprias e as farinhas que o dito Snõr governador lhes manda dar pera matolotaye estão no Cabo C.m pede a vosa merse os mande prover de farinha soleçeçinomente ate teren Rosarias de que comão $\mathbf{E}$ per ora lhas mande dar hus quinhentos Alqueires de farinha pera que senão espalhen e se vão a busquar de comer a outras partes desenparando aquele lugar contra o intento do Snôr governador he de Sua magestade. Resebera merse. C.m Avaliense os quinhentos Alquers de farinha que os Reverendos padres peden em vertude da provisão do snõr governador geral que para iso ten e Esta Registada a folhas sento e coatro na 
bolta ate sento e sinco no livro do almoxarife luis de mejreles barboza $\mathrm{E}$ da contia se lhe pase mandado para o Almoxarife sinco de dezenbro de seissentos $\mathbf{E}$ dezasete anos, Bulhão - treslado da provisão dos padres da Conpanhia -

Dom Luis de Sousa do Conselho de Sua magestade Senhor da vila de biringel. alcaide mor da sidade de beya. governador e capitan geral deste estado do Brazil Ett. a faso saber a vos francisco cabral provendor e contador da fazenda do dito Snõr na capitania do Rio de janeiro ou a quen tal cargo servir que sendo Sua mag.de informado do muito que conven a seu serviso provoar se 3 Cabo frio pera prevenir os intentos de alguas nasoins do norte, que frequentan aquela pazaien por causa da carrega do pao brazil ordenou o governador gaspar de Sousa meu antesesor que acodise a Remedear este dano pela maneira que lhe paresese e per quoanto os avizos e Reseos dos // enemigos se forão aveniudo mais per causas que sobrevierão me encarrega, agora. Sua mag.de a minha partida per sua carta que fica tresladada no Livro dos Resistos das provisõins siculares desta capitania de pernáobuoco a folhas sento e sincoenta $\mathrm{E}$ nove que con posivel cuidado e diligencia na forma da instrusão que para iso me mandou dar tratase de povoar o dito cabo frio per ser de importansia que se dejxe vir per conservasão das capitanias do Sul que ficaõ mui aRisquadas acontesendo que os estrangeiros contenuen com mais forsa a carga do dito pao brazil e por que o meo prinsipal que se Representa para este dano se poder Evitar he poar (sic) o dito cabo frio he situar nele duas Aldeas de indios de que taben o dito gaspar de sousa avija ja tratado ordenãodo ho o Capitão mor da capitania de São Visente mandase serto numero de cazais para ese hefeito he ora para os ditos indios se moveren. com mais fasilidade, yren Residir no dito cabo frio vão en hua enbarcasão que mando fretada pera ese efeito dous Religiozos da companhia com os mantimentos e Resgates nesesarios que os aconpanhen e leven a sua conta ej por ben e vos mando que pedindovos os ditos Religiozos $\mathbf{E}$ sendo the nesesaria coalquer outra couza para o hefeito sobredito lhe mandeis dar per conta da fasenda de Sua mag.de pasando mandado sobre o almoxarife desa capitania pela coal esta minha provisão que vira inserta nele. he sera tresladada no livro da despeza do dito Almoxarife $\mathrm{E}$ conhesimento das partes que Reseberen o que asin mandardes entregar lhe sera levado en conta a contia que nisto montar pelos contratadores deste dito estado sen enbargo da folha e das clauzulas dela e lhe dareis outro sy toda 
a ajuda e favor. para que Realmente se afeitue o que per esta minha provisão ordeno e per coanto parte dos ditos indios se ão de alevantar na capitania de São Vicente como dito he o provedor da fasenda dela conforme a ordem que the inviey ade acodir na mesma maneira aos ditos Religiozos con a despeza que alen das cousas sobre ditas lhe for nesesaria $E$ pode bem aconteser que a fasenda de Sua Mag.de na dita capitania esteya falta. vos mando que posandovos percatorio o dito provedor $\mathrm{E}$ pelo coal nos pesa ou outra coalquer couza para este hefeito vindo no dito precatorio inserta. a provisão que lhe pasej // the acudais con tudo o que asin vos pedir pasando como dito he mandado sobre o almoxafire desa dita capitania he sera obrigado ha cobrar pera sua conta conhecimento en forma da dita capitania de São Visente. pelo coal e o dito precatorio he a noso mandado lhe sera feita despeza do que niso montar dado en olinda sob meu sinal $\mathrm{E}$ selo de minhas armas aos dezasete. de fevereiro de mil he seissentos $\mathrm{E}$ dezasete anos o Governador don luis de Souza, o coal treslado de provisão fiz aqui treslador do livro a coal en todo $\mathrm{E}$ per todo me Reporto E vaj na verdade Eu Rafael de Carvalho a fis Escrever E sob escrevj Rafael de Carvalho.

E sendo en os dezanove dias do mes de Dezembro da dita era atras declarada o Capitão Rui vas pinto pasando pela porta dalfandega chamara o provedor dalfandega di.o Lopes de bulhão e andando paseando hum pedaso de tenpo chegou o provedor agastado a alfandega aonde tomou hun Livro dos Registos de luis de meireles he deu a min provisão digo escrivão hua provisão que no dito seler e desenbargadores se não metão en nenhua couza livro esta treladada. en que Sua mag.de manda ao chanseler e desenbargadores se não metão en nenhũa couza que toque a sua fazenda he dela dependa percoanto a Juis conpetente p.ra ella $\mathbf{E}$ mandando lha notifiquar per min escrivão con suspensasão de meu ofisio se não metese no tocante a fazenda do dito snõr e querendo lha eu escrivão comesar a ler me mandou prender pelo meirinho desta sidade e não quis que lese a provisão dizendo ao dito provedor per lhe diser avia de goardar seu Regimento o avia de mandar prezo ao Rejno e que se não metese en querer tomar farinha nen enbarcassão perque o não avia de consentir, he o dito provedor lhe Respondeo que avia de obedeser a provisão do s.r don luis de Sousa governador geral deste estado pois the vynha Remetida $\mathbf{E}$ avia de dar a ezecusão como nela lhe mandava per coanto a dita provisão não tratava con hele dito Capitão mor e ser a nesesidade da farinha muj urgente $\mathrm{E}$ pre- 
fis este termo per mandado do dito provedor o que sertifico pasar na verdade $\mathrm{E}$ o dito provedor tomou teste. munhas. de como o dito capitão mor lhe impidia o serviso de Sua magestade. e negosio de tanta importancia como era dar socorro aos indios Eu Rafael de Carvalho escrivão dalfandega $\mathbf{E}$ almoxarifado o hescrevi Rafael de Carvalho.

$1 /$

\section{Sertidão}

Certifiquo Eu Rafael de carvalho escrivão dalfandega e almoxarifado desta sidade de São Sebastiáo do Rio de Janeiro que e verdade que estando servindo de provedor da fazenda de Sua mag.de Diogo lopes de bulhão per provisão do dito Snõr que o dito $s r$ governador geral do estado do brazil Don luis de Sousa lhe escreveo huma carta en a coal vinha hum Capitolo cuio treslado e o seguinte, con o Capitão mor Rui vaz pinto tenha Vosa merse toda a boa conRespondensia tratando que se não exseda no numero das prasas que vão na folha e que esas seyão heffectivas e que os soldados e ofisiais cumpráo con suas obrigasoiñs na parte aonda Resediren fazendo listra de tenpo en tenpo para ver o como serven e os que faltão, $\mathrm{E}$ o dito capitulo de carta não dis mais a coal me Reporto e En conformidade do dito capitulo o dito provedor da fazenda mandou pelo meirinho dalfandega fransisqo da costa que noteficasen aos soldados que andavão nesta sidade. se Recolhesen as suas fortalezas e que não no fazendo asi se lhe não faria pagamento. da fazenda de Sua mag.de e logo no dito dia o Capitan mor Rui vas pinto mandou chamar a min escrivão que Requerese de sua parte ao dito provedor da fazenda que se não intremetese. con os saldados das fortalezas que erão de sua Jurisdisão con penas de quinhentos cruzados $E$ suspensasão de seu cargo e de o enbarquar. para o Rejno prezo e não pera onde estava o governador geral. per que a carta que lhe escrevera não era couza de consyderasão he que fisese o dito Requerimento ao dito provedor com pena. de suspensasão de meu ofiçio e per me ser pedida a presente. a pasej na verdade Rio de janeiro quinse dias de novembro de seissentos e dezasete anos Rafael de Carvalho.

- outra sertidão -

Certifico eu domingos ferreira de brito alferes da fortaleza Sn.ta Crus da barra desta sidade do Rio de jan.ro por sua mag.de que e verdade pelo Juramento que Resebi de meu cargo que na fortaleza asisten somente. sete soldados he hum condestable. e o cabo descoadra e per 
asy pasar na verdade he esta mo ser pedida. lha pasej permin feita e asinada oie vinte e ojto de janejro de mil e seis sentos $\mathbf{E}$ dezojto anõs, Domingos ferrejra de brito.

3. ${ }^{\circ} .^{\circ} \quad / /$

$$
\text { - outra sertidão - }
$$

Certifico eu Clemente noqueira capitan da fortaleza. de São João que he verdade que na fortaleza asisten somente sinco soldados he hum bonbardeyro $\mathrm{E}$ per me ser pedida a prezente a pasej pello Juramento de meu cargo. oie trinta de dezenbro de mil e seissentos $\mathrm{E}$ dezoito anos: - o Copatião (sic) Clemente noqueira.

$$
\text { - outra - }
$$

Certifico Eu Sebastião Coelho damij escrivão do almoxarifado e alfandegua en esta sidade De são Sebastião do Rio de janeiro que e verdade he dou minha feeque despois que os mestres dos navios tem o despacho dalfandega andão des ou doze dias na sidade he se queixão. que o Capitão mor lhes não quer dar o despacho das fortalezas E per me ser pedida a presente a pasej ao provedor da fazenda diogo lopes de bulhão en seis de abril de seissentos E dezanove anos, Sebastião Coelho damin.

$$
\text { - outra - }
$$

Certifiquo eu baltezar da costa escrivão da fazenda. nesta sidade de São Sebastião do Rio de janeiro que achando me con o provedor da fazenda. na alfandega diogo lo-. pes de bulhão Resenseando contas ao almoxarife que foi Luis de meireles barboza dise o dito provedor lhe punha: duvida a sento e sesenta e tantos mil Reis de hum man-. dado de cousas que se derão ao Capitão mor $\mathbf{E}$ governador Ruj vas pinto pera a ida que fes ao Cabo frio he asy. mais En dezaseis prasas de soldados. E per asin pasar na. verdade e o dito provedor me pedir o prezente. a pasej no Rio de jan.ro en des de abril de seissentos $\mathrm{E}$ dezanove anõs - Baltazar da Costa - O qual treslado de pa-. peis atras Eu B.ar da Costa. escrivão da faz.da nesta ci-. dade de San Sebastian do Rio de jan.ro fis tresladar dos propiros q. tornei a parte ben e fielmente sen cousa q.. duvida faca no Rio de jan.ro oito de mayo de seiscentos. $\mathbf{E}$ dezanove annos.

B.ar da costa.

Concertado per min escrivã.

B.ar da costa.

E comigo Escrivão Rafael de Carvalho. 
A. H. U. - Rio de Janeiro - 1619.

Por infinittas vezes ttenho feitto sabedor a vmg.de no Conselho da fazenda dos Roubos que por estas parttes se fazem a Real fazenda de vmg.de Em espicial Em materia de pão brazil, E cadaves se farão mais com grande soltura - item - ho ouvidor q. foi desta Capp.tta ho L.do g.lo homen dalmeida per hordem dos governadores gaspar de Souza E dom luis de Souza, que me servem ttiram huns devassa ao qual $\mathrm{Eu}$ assistj a mor partte $\mathbf{E}$ ficaráo culpados Constantino de menelão cappittão a quem Eu vim susceder $\mathrm{E}$ ho Cappittão que Estao no Cabo frio estevão gomes com mais oito ou des pesoas desta Capp.tta E vindo ho desEmbargador anttão de misquitta per hordem do g.dor ttirar Rezidençia ao ditto constantino de menelas E tratar da Reformação da just.a que lhe foi Entregue a devaça per hordem que p.a isso trazia ho qual tratou destas couzas En forma que os culpados ficarão sem castigo $\mathrm{E}$ ho ouvidor que tinha ttirado a devassa com m.ta cristandade inteireza $E$ bom zelo do serviço de vmg. de, ficara pagando peccados alheos porque na Rezidencia que tambem the tirou ho desEmbargador chegou Constantino de menelão alguas test.as a seu modo que jurarão que ho dito ouvidor induzira test.as na devasa do pão $1 .^{\circ}$ v. ${ }^{\circ}$ brazil mil ttraças $\mathrm{E}$ modos se buscarão p.a vgm.de ser mal servido, estando sempre neste ttempo // Constantino de menelao $\mathrm{E}$ ho desEmbargador $\mathrm{Em}$ braços $\mathrm{E}$ desejou ttantto salvalo que ho deixou hir correndo has Cappittanias do Cabo frio Spiritu Sancto E portto Seguro na qual deixou comprado hum Engenho p.a se agazalhar ahi ho que Entendo não ser serviço de vmg.de $E$ ha dias que Esta na bahia esperando pela Rezidençia que ficou Em poder do desEmbargador por não ser vista nem sentenseada sem elle estar prezentte. - item - hum almofaris que avia nesta sidade que tinha des ou doze quintais de mettal, Em que se fazia polvora E hum signo que Estava na fortaleza dela que servia aos Rebattes e escuzava os gastos de polvora que se agora fazem $\mathrm{E}$ asim mais muita artelharia de bronze com as armas Reais se avrigua desfazer ttudo constantino de menelao $\mathrm{E}$ a mor partte se desfes Em chumaseiras p.a, os Engenhos Em que se fes: $\mathbf{m}$.to dinheiro $\mathbf{E}$ disto não ttratou ho desEmbargador sendo nottorio a thoda a tterra que o ditto consttantino de menelao comettera $\mathrm{E}$ fizera este delicto - item - quizera devasar dese negoçio, mas temi que me levantasem que induzia ttestemunhas, pelo que ordenei da parte de vmg.de ao provedor da fazenda ttirasse esta devassa E persuadido o fes estando a ttudo prezentte hum paulo Rodrigues homem de nação Em cuja casa se fundio a artte- 
lharia $\mathbf{E}$ mais cauzas a quem ho governador dom Luis de Sousa passou hua provizão p.a servir como serve de procu. rador da coroa e fazenda devia ter falça informação porque ho governador $\mathrm{E}$ zelozo do serviço de vmg.de faltão desassette pessas de artelharia dous mil pelouros dela, que ttodos se disfizerão, que ttantos $\mathrm{E}$ mais faltão sem aver ocazião de gerra nem se gastar nisso hum soo, vmg.de se for servido // mande acudir a isto $\mathrm{E}$ a outras muitas couzas que ho desEmbargador ttrattou soo do que lhe pareseo $\mathbf{E}$ não do serviço de vmg.de $\mathbf{E}$ soo por que dele the fazia advirtençias me tomou ttamanho odio que he meu imigo cappittal E se foi sem me falar - Suas couzas estão mal Reçebidas na Relação por que m.tas que de pottençia aqui quis fazer de que se agravou dele vierão ttodas providas. item - Até oiê não ttive cartta nem Recado de vmg.de mas Espero que no primr.o navio venha ordem Em que vmg.de me faça $\mathrm{m}$. mandar me ttomar Rezidencia $\mathrm{E}$ levantar a omenajem que desta capp.tta ttenho dado pela Rezois de que ja avizey a vmg.de $\mathrm{E}$ cada dia cressem mais por que não sofro que se sirva mal a vmg.de aonde $\mathrm{Eu}$ estou. item - $\mathrm{Da}$ fazenda de vmg.de despendi por ordem do g.dor geral ho que consta da serttidaõ junta $\mathrm{E}$ soo por minha ordem tenho gastado da Real fazenda por hua vez tres mil Reis $\mathrm{E}$ doze alqueires de farinha de gerra por outra como ttodo se ve da mesma sertidão e os mais capittais que tem sido nesta cappittania por sua ordem despenderão sempre dous tres mil cruzados da faendza de vmg.ed sem se avizar que fazião despezas $\mathrm{E}$ eu de quoatro mil $\mathrm{E}$ duzentos Reis que mandei despender forão queixas a vmg.de que Eu fazia extraordinarias saidas sem nesescidade soo pera despender, As que $\mathrm{Eu}$ intentei fazer foi por acudir ao serviço de vmg.de E dar na ilha de Santa anna Em naos que ahy estavão carregando pão Brazil $\mathrm{E}$ posto que fis os gastos por contta de minha fazenda // buscarão ttodos os meos p.a se me impedir a viajem $\mathbf{E}$ ho desEmbargador com grande instançia aperttou com os vereadores $\mathbf{E}$ povo que me fisesem Requerimentos que não saisse pela barra fora não sei seu intento mas soo sei que as naos carregarão muito a seu salvo $E$ se forão Embora podendo se lhe attalhar noso Snõr a Real E catolica pessoa de vmg.de gr.de Rio de janr. de junho de 619 a

Rui Vaz pinto.

A margem: Rio de Jan.ro -8 de Junho de 619 .

Carta de Ruj vaz pinto Cappt.ão do Rio de Jan.ro sobre matt.rias da faz.a. 
A. H. U. - Rio de Janeiro - 1619 .

Snõr.

SuPosto que tenha escrito a vmg.de sobre esta materia são tantas as ocaziões que de novo cada ora sucedem que he a cauza de aguora o tornar a fazer com novas queixas do caPitão mor desta sidade Ruj vas pinto o qual Posto que do g.dor geral deste estado he de ordinario RePrendido das desordeñs que fas. nem Por iso eixa de ir com elas avante e cada ves em mayor cresimento atalhando me e emPedindo com Potensias e termos desuzados a que Posa inteyramente comResponder com a obriguação do carguo de Provedor da faz.da Real de vmg.de de que me fes M. emcarreguar e Para q. de tudo conste com serteza emvio a vmg.de os paPeis que com esta serão os quais Peso de M. mande vmg.de ver no tribunal de sua fazenda e mandar me a que nzais for do serviço de vmg.de dando a estes Particulares o Remedio que vmg.de for servido cuja Catholiqua e Real Pessoa g.de des ett.a Ry.o de jan.ro 13 de dezembro de 1619.

Dioguo lopes de bulhão.

No verso: Rio de jan.ro - 13 de Dez.ro 619.

Do Prov.or fa f.a daquella Cap.a

Sobre o mao proçedim.to q. teve Ruj vas pinto Capitã daquella prassa.

*

A. H. U. - Rio de Janeiro - 1619 - Doc. anexo.

Dioguo lopes de bulhão q. a ele the he nesesario o treslado dos papeis e autos q. com esta oferesse tornando se the os proprios por vias

p.a Vm lhe fasa m. mandar lhe dar os ditos treslados en modo q. fasão fe por hu ta.m e Rm.

Como pede Rio de Janr.o 9 de dezbr. de 619

Estevão daraujo ttreslada do pedido -

Auto que mandou fazer o provedor da faz.da de sua mag.de Diogo lopes de bulhan.

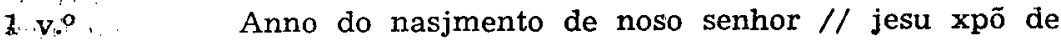
mil seissentos $\mathbf{E}$ dezanove annos aos vinte e nove dias do mes de agosto da dita Era nesta cidade de sam Sebastião do Rio de Janeiro na alfandegua dela estando ahi o Provedor da fazenda de sua magestade dioguo lopes de bulhan por Elle foi mandado fazer este auto dizendo que 
por quoanto o caPitan $\mathrm{E}$ guovernador desta dita cidadeRuj vas Pinto se entremete por alguas vezes em couzas ttocantes a fazenda de sua mag.de $\mathrm{E}$ dos ofisiais della não. tendo pera iso jurisdisão algua the mandou notefiquar hua provizão do guovernador gueral deste estado Dom Luis de souza em que manda ao dito Capitam e g.dor se. não jntremeta por nenhua via em couzas ttocantes a fazenda do dito snõr que estam a carguo delle dito provedor a quoal notefiquasão the foi feita por min escrivão lendo. lhe a dita provizão de verbo ad verbu ficando the em. seu poder o treslado della aos dezoite dias destte prezente mes de agosto $\mathrm{E}$ anno $\mathrm{E}$ despois da dita notificasão sendo em os vinte e oito do dito mes mandou no // ttefiquar a dous mestres de navios que estam no porto desta cidade vindos do Reino com fazendas a ella a saber manoel pires E lionardo vas con çertas Penas não não (sic) trouxesen as fazendas a esta dita alfandegua tendo comesado o dito lionardo Vaz a descarreguar $\mathbf{E}$ despor dandolhe niso muita molestia tudo contra forma de hu capitolo de seu Regimento em que sua magestade manda ao dito capitáo mor fasa bon tratamento aos mestres dos navios que vieren de fora a este portto $\mathrm{E}$ outro capitolo do dito Regimento em que o dito s.or lhe manda deixe livremente servir a seus oficiais da fazenda seus cargos sendo asi mais que o dito Capp.tam e governador nomea por goardas dos navios criados seus que tem matriculados por soldados os quoais vão fazer o dito ofiçio de goarda sen nenhum Provimento nem juram.to de que naçe sahirense dos ditos navios cada ves que querem e virem pasear a terra como foi antonio da silva criado seu que meteo Por goarde no navio de fr.co Luis he estando nele sahio por vezes que foi visto pasear nesta cidade com costa de hum auto $\mathrm{E}$ jmformasão que diso se fes he prera (sic) que cnoste (sic) das noteficasões // feitas aos ditos mestres $2 \mathrm{v}^{\circ}$ mandou que viesen diante delle pera com juramento declararem ser lhes feita as ditas noteficassois de que fes: este auto $\mathrm{Eu}$ fran.co da Costa escrivão da provedoria da fazenda o escrevj - Diogo lopes de bulham -

Aos ttrinta dias do mes de agosto deste prezente anno de sseissentos $\mathrm{E}$ dezanove eu escrivão com o Provedor da fazenda de sua magestade ttiramos as testemunhas ao diante nomeadas pello autor atras na alfandegua desta cidade $E$ seus dittos são os que ao diante se seguem eu fr.co da Costa escrivão da provedoria da fazenda o escrevj item Lionardo Vaz ttestemunha a que o Provedor deu juramento dos santtos avamguelhos em que pos a mão pro- meteo dizer verdade he de idade de trinta $\mathbf{E}$ tres annos pouquo mais ou menos $\mathrm{E}$ do costume dise nada. 
$3 r^{\circ}$

$-3 \vee \circ$

(a). - na entrelinha.
Perguntado Pello conteudo no auto atras que lhe foi lido dise elle test.aa que aos vinte $\mathbf{E}$ oito deste prezente mes pello escrivão antonio pimenta dabreu // lhe foi feita hua notefiquasão da parte do Capitam $\mathrm{E}$ guovernador Rui vaz pinto que não descarreguase fazenda algua "na alfandegua" (a) por quoanto tinha mandado notefiquar ao provedor Diogo lopes de bulham que mudase a dita alfandegua $\mathrm{E}$ al não dise $\mathrm{E}$ assinou con o dito Provedor Eu fr.co da Costa escrivão da provedoria da fazenda o escrevj - Lionardo Vaz - Dioguo lopes de bulham -

Aos trinta e hum dias do mes de agosto do anno atras escrito na alfandegua desta çidade eu escrivão com o provedor da fazenda de sua magestade dioguo lopes de bulham tiramos as test.as ao diante nomeados (sic) pello auto atras eu fran.co da Costa escrivão da provedoria da fazenda o escrevj - item Pantalião pinto estante nesta sidade mestre da nnao nomeada nosa S.ra da boa ora testemunha a quem ho provedor deu juramento dos Santos Evão-guelhos em que pos a mão prometeu dizer verdade he de idade de vinte annos pouquo mais ou menos e do costume dise nada - // Perguntado pello conteudo no auto atras dise elle testemunha que he verdade que o Capitam mor Ruj vas pinto lhe mandou notefiquar que não trouxese fazenda algua a alfandegua ate não estar fechada $E$ al não dise $E$ asinou cõ o dito Provedor eu fran.co da Costa escrivão da fazenda e provedoria o escrevj Pantaleão pinto - Diogo lopes de bulham -

item Manoel pires estante nesta cidade senhorio de nnao nomeada nossa 'senhora do Rozairo test.a a quem foi dado juramento dos Sanctos avamguelhos em que pos a māo prometeu dizer verdade he de idade de quaremta annos pouquo mais ou menos $\mathbf{E}$ do costume dise nada -

Perguntado pello conteudo no autor attras dise elle testemunha que he verdade que o Capitam mor Ruy vaz pinto the mandou notefiquar que não tirase nem desembarquase fazenda algua sem primeiro aver outra alfandegua $\mathrm{E}$ al não dise $\mathrm{E}$ asinou con o dito provedor eu fr.co da costa escrivão da pro // vedoria da fazenda o escrevj - Manoel pires - diogo lopes de bulham -

Sertefiquo eu fran.co da costa escrivam da Provedoria da fazenda nesta cidade de sam Sebastião do Rio de janeiro por Sua magestade que aos vinte e sete dias do mes de julho deste prezente anno de seissentos E dezanove Estando o provedor da fazenda Diogo lopes de bulham pera fazer audiençia veo hum homem de caza do caPitam $\mathrm{E}$ guovernador Rui vas pinto a Dita alfandegua $\mathrm{E}$ de sua 
parte dise ao dito provedor que chegase a sua caza que. competia asin ao serviso de sua magestade o que logo o dito Porvedor fes jndo eu escrivam Em sua companhia E: o meirinho do mar $\mathbf{E}$ o almoxarifee desta çidade e chegando a sua caza lhe dise que sua merçe o mandara chamar de parte de sua magestade ao que obedesia e por acodir loguo ao que fose nesesario deixara de fazer audiensia e pello dito g.dor foi dito que os mezes pasados vindo lhe a nnoticia que desta cidade faltava algua artelharia do dito snõr lhes requerera tirase diso devasa $\mathbf{E}$ que ategora lhe não tinha dado conta do que niso fizera nem the dera Rol dos culpados // pera os prender e o dito provedor rrespondeo que elle tirara a devasa $\mathrm{E}$ a tinha Remetida ao s.or Governador. geral deste estado E provedor mor por lhe Pareser mais serviso do dito senhor $\mathrm{E}$ o dito Capitam E governador Ruj Vaz pinto lhe asinou tres dias pera que dentro nelles pernunciase a dita devasa $\mathbf{E}$ culpados della e lhe dese o Rol delles $\mathrm{E}$ que $\mathbf{f a -}$ zendo o contrario proveria o dito carguo de provedor em outra pesoa que o fizese $\mathrm{E}$ em Ressolusão lhe Respondeo. o dito provedor que elle tomaria ssobre iso concelho con. os letrados que avia na tterra $\mathrm{E}$ conforme iso faria o que fose mais sserviso de sua magestade $E$ bem de sua fazenda $\mathbf{E}$ por pasar na verdade pasei esta por mim feita $\mathbf{E}$ asinada em Rio de Janeiro vinte $\mathbf{E}$ nove de julho de mil sseseiscentos $E$ dezanove annos - fr.co da Costa barros -

$5 \mathrm{r}^{\circ}$. Sertifiquo eu fr.co da Costa escrivão da Provedoria da fazenda nesta cidade de sam sebastião do Rio de Ja-. neiro por Sua magestade que tendo o capitam // $\mathbf{E}$ governador Ruj vas pinto Duvidas cõ o Provedor $\mathrm{Da}$ fazenda diogo lopes de bulhão ssobre huas pipas de fazenda que tinham vindo De Angola dizendo o dito Provedor que as mandava vir a alfandegua pera nellas seren vistas E despachadas o dito Capitam mor o Refuzou dizend.y que as fosen ver ao proprio navio $\mathrm{E}$ não querendo dito. Provedor vir a iso por dizer que o Regimento não Dava lugar senão pera as ditas fazendas hiren direitamente a. alfandegua o dito Capitam dise que mandaria chamar hum juis $\mathbf{E}$ as hiria ver $\mathbf{E}$ abrir ao dito navio $\mathbf{E}$ Despois de o dito Provedor estar na alfandegua tendo mandado ao meirinho do mar fr.co da Costa que lhe trouxesse a ella as ditas fazendas estando eu escrivam prezente chegou hum criado do dito capitam mor $\mathrm{E}$ de sua parte diçe ao dito Provedor que lhe fizese merce de não bulir nas dittas fazendas $\mathrm{E}$ de as deixar estar na forma que estavão E o dito Provedor Respondeu que não podia deixar de as mãdar vir $E$ despachar o que tudo sertifiquo por me achar Prezente em o Rio de janeiro trinta de julho de mil e. 
seissentos E dezanove annos - fran.co da Costa baRos // Sertifiquo eu franc.o da Costa escrivão da provedoria da fazenda nesta cidade de sam sebastiāo do Rio. de janeiro que aos vinte e dous dias do mes de agosto deste Prezente anno de seissentos $\mathrm{E}$ dezanove Estando eu: escrivão com o provedor da fazenda Diogo lopes de bulham na alfandegua della pareseu o sargento mor Migel. montr.o E por elle foi dito que de parte do dito Provedor Ihe fora noteficado q. ajuntaçe os soldados de prezidio. desta dita çidade pera se fazer delles lista $\mathrm{E}$ se lhe pagar na forma do Capitolo da carta do senhor guovernador gueral E Provizão do Provedor mor Pero de gouvea de melo que tinha $\mathrm{E}$ que elle sargento mor se não metia niso nem. conheçia os ditos soldados pera o poder fazer $\mathrm{E}$ que jso. tocava $\mathrm{E}$ pertençia ao Capitam mor o que tudo pasa $\mathrm{na}$ verdade $\mathrm{E}$ o meirinho Do mar fran.co da Costa me deu por fee fazer a dita noteficasão ao dito sargento mor de que Pasei a prezente no Rio de janeiro vinte e tres de. agosto de mil seissentos $\mathrm{E}$ dezanove annos $\mathrm{E}$ declaro que pasei outra deste teor sobredito o escrevj fr.co da Costa. barros -

6 r. $^{\circ}$ Certifiquo Eu fran co da Costa escri // vão da Provedoria da fazenda nesta cidade de san sebastião do Rio de janeiro por sua magestade que despois que sirvo o dito carguo sei que sempre o Capitão he guovernador Rui vaz pinto teva má vontade ao provedor da fazenda dioguo lopes de bulham $E$ asin o manifestou em ttodas as ocaziões he em espesial depois que o dito Provedor diçe. que não avia de fazer pagamento a soldado nenhum salvo aquelles que fosem efectivos mostrãdo pera iso hum Capitolo de hua carta do guovernador gueral deste Estado dom luis De souza e hua Provizão do provedor mor pero de gouvea de melo em q. Ihe ordenão que não mande paguar senão Pella dita manejra de que tem Rezultado dizer o dito capitam mor publiquam.te que o ade suspender $\mathbf{E}$ mandar prezo em ferros a Portugual antremetendose $\mathrm{Em}$ sua jurdisão nas materias da fazenda $\mathrm{E}$ por me ser pedida a prezente de meu oficio a pasei por min asinada no Rio de jan.ro vinte $E$ nove de agosto de seissentos E dezanove annos - fran.co da Costa barros -

6 v. ${ }^{\circ}$ Sertifiquo eu sebastião coelho damŭ es // crivão do. almox.do e alfandegua nesta cidade de são senastião Rio de janeiro por sua magestade que depois que sirvo o dito. carguo sei sempre o capitam $\mathrm{E}$ guovernador Rui vas pinto theve ma vontade ao provedor da fazenda Diogo lopes de bulham he asi o manifestou en todas as ocazois he em espesial depois que o dito provedor dise que não avia de fazer Pagamento a soldado nenhum salvo haquelles que 
fosen efetivos mostrando Pera iso hum Capitolo de hua carta do guovernador gueral deste estado Dom Luis de souza $\mathbf{E}$ hua Provizão do provedor mor Pero de gouvea de melo em que the ordenam que não mande pagar senão Pella dita maneira de que tem Rezultado dizer o dito Capitam mor publiquamente que o ade suspender e mandar prezo em ferros a portugal emtremetendose em sua jurdisão nas materias da fazenda he por me ser pedida a prezente de meu oficio a Pasei por min asinada nno Rio de jan.ro vinte $E$ nove de agostto de seis sentos $E$ dezanove annos - ssebastião Coelho damĭ -

7 r. $\quad$ Sertefiquo eu fran.co da Costa escri // vão da fazenda nesta cidade de sam sebastião do Rio de janeiro por sua magestade que as cazas que de prezente servem Dalfandegua nella sam as milhores que atee agora ha avido em sitio fortaleza $\mathbf{E}$ en tudo o mais que se Requere Pera bom despacho $\mathrm{E}$ aviaments das fazendas que a ella vem $\mathrm{E}$ por me ser pedida a Prezente Pello provedor da faz.da de sua mag.de diogo lopes de bulham passei ha Prezente por mim asinada nesta dita cidade Em simquo de Dezembro de mil $\mathrm{E}$ seissentos e dezanove annos - fran.co da Costa barros -

Auto feito Por mandado do provedor da faz.da Diogo lopes de bulhã. Anno do nasjmento de noso s.or Jesu xpõ $\mathrm{De}$ mil $\mathrm{E}$ seis sentos $\mathrm{E}$ dezanove annos aos ttrinta $\mathrm{E}$ hum dias do mes de agosto da dita era nesta cidade de são sebastiáo do Rio de janeiro na alfandegua della estando ahi o Provedor da faz.da diogo lopes de bulham por elle foi dito que o capitam $E$ governador Rui vas pinto contra seu Regimento E o desta alfandegua se emtremetia Em sua jurdi // ssão he o Perturbava $E$ inquietava nela mandando vezitar os navios que neste Porto entrão pondo nelles goardas criados sseus sem provimento algum nem juramento $E$ impedindo que elle provedor $o$ não faça dando ocazião a muitos descõssertos e a que os oficiais da fazenda nnão posão bem fazer seus ofiçios pera Prova do quoal mandou a min escrivão que aqui lancase minha fee $\mathrm{E}$ acostace os autos que do sobredito sse tem feito $E$ que outrosin por elle provedor fazer exame no tocante aos soldados de prezidio que sua magestade manda aja nas fortalezas desta cidade obrigandoos $\mathrm{E}$ constrangendoos a que sirvão nellas lhe ten cobrado odio $\mathbf{E}$ $0^{\prime}$ ameassou por muitas vezes dizendo que elle dito Provedor não tem poder nnen orden Pera conheser diso $\mathbf{E}$ do que elle fizer nesta materia ninguen lhe Pode ttomar conta senão el Rej ssem embargo de elle dito Provedor lhe ter mandado notefiquar hua Provizão E hum capitolo de hua carta do guovernador gueral deste estado Dom Luis 
de souza $\mathrm{E}$ outra provizão do provedor mor Pero de gouvea de mello em que the mandão que de nhua manejra se i8r. ${ }^{\circ} / /$ Entremeta nas couzas da fazenda de sua magestade $\mathrm{E}$ a elle provedor que não pague senão aos soldados que serviren efetivamente pera o que da em prova as ditas Provizõis ou treslados dellas con as fes das noteficasois $\mathrm{E}$ ora novamente mandando o dito Capitão mor tirar a folha dos ditos soldados bombardeiros $\mathbf{E}$ mais oficiais das fortaleazs em que elle Provedor asinou Pera se lhe fazer pagamento de seus soldos por ser nesesario fazer ssobre iso Particular exame por lhe cnostar (sic) que alguns dos nomeados na dita folha não tinhão merecido soldo nen tinhão servido conforme o alvara de sua magestade $\mathrm{E}$ as ordens do dito guovernador gueral $\mathrm{E}$ provedor mor mandando notefiquar ao almoxarife felipe fr.o não fizese pagamento a soldado nenhum sen sua ordem o dito Capitam mor o mandou suspender como constara da fee do tabelião antonio Pimenta dabreu que de sua Parte lho noteficou com q. fee do meirinho do mar fran.co da Costa mandando outrosin noteficar $\mathbf{E}$ noteficando pesoalmente aos mais oficiais da fazenda que com pena $/ / \mathrm{De}$ ssuspensão de sseos oficios não servissem mais con elle dito Provedor dizendo que o avia de embarcar prezo em ferros e avia de prover provedor que Paguase aos soldados que publiquamente andão nesta cidade paseando $\mathbf{E}$ muitos ttrabalham por seus oficios $\mathrm{E}$ servem oficios de justisa $\mathrm{E}$ de goardas de navios sem jrem as fortalezas como sua magestade ordena que sirvão $\mathrm{E}$ botando pregois por ttoda esta cidade con caxa ttocada que toda a pesoa que quizece servir nas fortalezas de soldado se fose a Prezentar na camara pera se matricular nos livros della E fazendo outros muitos excesos ssoo a fin de molestar a elle dito Provedor $\mathbf{E}$ de lhe querer uzurpar $\mathbf{E}$ desipar sua jurdisão $\mathbf{E}$ de despender a fazenda de Sua magestade por seus criados a que tten nomeados por soldados $E$ he publico $E$ notorio por toda esta cidade que por estte Respeito $\mathrm{E}$ de elle dito Provedor defender a fazenda Real lhe fes o dito capitan mor as ditas molestias $\mathrm{E}$ ordenou as couzas ssobreditas de que tudo mandou fazer este auto pera que a todo tempo conste do sobredito Em que asinou E pera prova delle mandou ha // min escrivão que de

9r..$^{-}$thodo o conteudo nelle lansase minha fee e lhe ajunttaçe os autọs sertidois $\mathbf{E}$ mais papeis nelle declarados fr.co da Costa escrivam da Provedoria da fazenda o escrevj dioguo lopes de bulham -

Satisfazendo com o que me he mandado nno auto asima $\mathbf{E}$ atras sertifiquo eu fran. co da Costa escrivão da provedoria da fazenda nesta cidade de são sebastiāo do 
Rio de janeiro que do tempo que sirvo o dito oficio sei que o capitan e g.dor Rui vas pinto tem posto e poem nos navios que a este porto vem goardas criados seus $\mathbf{E}$ ate oie não vi Provizão porque sirvão os ditos oficios nem: nesta alfandegua $\mathbf{E}$ provedoria se deu ate agora juramento a nenhum $\mathrm{E}$ he publiquo $\mathrm{E}$ manifesto mandar vezitar os 9v. ${ }^{\circ} \quad$ navios por criados seis $\mathrm{E}$ pesoas que lhe Parece $\mathrm{E}$ tem mandado ao meirinho do mar fran.co da Costa que não va aos ditos navios quoando Entrão E. en tudo me Reporto aos autos que diso estam feitos $\mathrm{E}$ outrosi sei que vindo a este porto hum navio de buenos aires mandou // o Provedor da fazenda Dioguo lopes de bulham a elle hum goarda $E$ o dito caPitam mor não conçentio que fose a elle antes mandou ao goarda que logo se tornaçe $\mathbf{E}$ no tocante aos soldados sej que o dito Provedor fas $E$ ten feito exames $\mathrm{E}$ imformasois $\mathrm{E}$ ten mandado ao almoxarife felipe fr.a não pague aos que não servĕ nas fortalezas comforme o alvara del Rej noteficando lhe hua provizão do provedor mor em que manda se não faça o dito Pagamento senão aos que efetivamente servem $\mathrm{E}$ he publiquo por toda esta cidade que sobre o cazo o dito Capitão mor o mandou suspender $\mathbf{E}$ mandou notefiquar aos oficiais da fazenda não servisen con elle com pena de suspensão de seus oficios $\mathrm{E}$ o ameasa publiquamente dizendo que o ade mandar prezo em ferros he que ningen lhe ade tomar diso conta senão el Rej $\mathrm{E}$ en tudo me Reporto a outtras sertidois $\mathbf{E}$ fees que do sobredito tenho Pasado no Rio de janeiro ttrinta $\mathbf{E}$.hum de agosto diguo promeiro de setembro de seissentos $\mathbf{E}$ dezanove annos franc.o da Costa barros -

10r. 0 O senhor Capitam $\mathbf{E}$ guovernador // Ruj vas pinto memandou "chamar a sua Caza E sendo la me mãdou" (a) que fose com com o meirinho fran.co da Costa a caza do Provedor Diogo lopes de bulham $\mathrm{E}$ de sua parte dizerlhe que o avia por suspenso ate merce Del Rei ou do senhor guovernador gueral por jmpedir ho serviso de sua magestade hua e m.tas vezes e elle o não poder fazer con elle $\mathrm{E}$ de como asi mo mandou pasei a prezente pedido Pello dito Provedor em que asinei con o dito meirinho fran.co da Costa oje trinta $\mathrm{E}$ hum de agosto de seissentos: $\mathbf{E}$ dezanove annos - anttonio pimenta dabreu - fr.co da Costa.

Sertifiquo eu fran.co da Costa escrivão da fazenda nesta cidade de san sebasttião do Rio de Janeiro por sua magestade que aos trinta $\mathbf{E}$ hum dias do mes de agosto deste prezente anno de seissenttos $\mathrm{E}$ dezanove sendo en--

(a). - na entrelinha. 
trado no porto desta dita cidade hum navio do Rio da prata $E$ buenos aires de que he mestre joão mez farello mandando o provedor da fazenda Diogo lopes de bulham a elle por goarda a antonio gomes con juramento que Ihe tinha dado $\mathbf{E}$ con hum escrito seu sabendo o Capitan

10v..$^{-E}$ governador Rui vas // Pinto que hia o dito goarda o chamou e lhe ttomou o escrito que levava $E$ the mandou que não fose ao dito navio $\mathrm{E}$ lloguo mandou Pello taballião antonio pimenta $\mathbf{E}$ Pello meirinho do mar fran.co da Costa que em nome de sua magestade suspendese ao dito Provedor ate ordem do dito senhor e "do" (a) guovernador gueral Dom Luis de souza $\mathbf{E}$ por pasar na verdade $\mathbf{E}$ me sser pedida a Prezente de meu oficio a Pasei por mim asinada no dito dia asima franc.o da Costa barros -

Sertifiquo eu sebastião coelho dami escrivão do almoxarifado $\mathrm{E}$ alfandegua nesta cidade de sam sebastião Rio de janeiro por sua magestade que aos trinta $\mathbf{E}$ hum dia do mes de agosto deste prezente anno de seissentos $\mathbf{E}$ dezanove sendo emtrado no porto desta dita cidade hum navio do Rio da prata $\mathrm{E}$ buenos aires de que he mestre joão martins farello mandando o Provedor da fazenda Dioguo lopes de bulham a elle por goarda a antonio gomes con juramento $\mathrm{q}$. The tinha dado $\mathrm{E}$ con hu escrito seu sabendo o Capitam $\mathbf{E}$ governador Rui vas pintto que hia o dito goarda o chamou e lhe tomou // o escrito que levava $E$ lhe mandou que náo fose ao dito 11r. ${ }^{\circ}$ navio $\mathrm{E}$ loguo mandou pello tabalião antonio pimenta e pello meirinho do mar franc.o da Costa que en nome de sua magestade ssuspendese ao dito Provedor ate orden do dito senhor $\mathrm{E}$ do guovernador gueral dom Luis de souza e por pasar na verdade $\mathbf{E}$ me ser pedida a prezente de meu oficio a pasei por mim asinada no dito dia asima - sebastião Coelho damin -

Auto que me mandou fazer o provedor da fazenda Diogo lopes de bulham. Anno do nasimento de noso senhor jesu xpõ de mil $\mathrm{E}$ seissentos $\mathrm{E}$ dezanove annos aos vinte e quoatro dias do mes de setembro da dita era nesta cidade de sam sebastião do Rio de janeiro na alfandegua della Pello provedor da fazenda Diogo lopes de bulham foi mandado fazer este auto pera por ello constar o seginte que o administrador desta Repartisáo Matheos

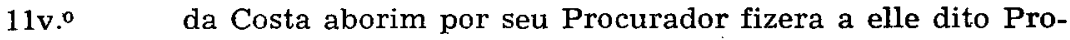
vedor Petisão // comforme hua Provizão de sua magestade dizendo que elle estava de caminho pera hir vizitar a Capitania de sam Visente pera o que lhe pedia lhe man-

(a). - na entrelinha. 
dase dar embarcasão E matalotagm neseçaria por vertude da quoal Provizão que esta Registada nos Livros do almoxarifado desta cidade mandara Pasar mandado pera o almoxarife feliphe fr.a dar a dita matalotagem $\mathbf{E}$ mandara notefiquar a lionardo vas mestre e senhorio do navi, nomeado nnosa s.ra do Rozario se fizese Prestes com o dito navio Pera ir levar ao dito admenistrador louvandose por sua Parte em pesoa que avaliaçe o frete dele $\mathbf{E}$ por o dito lionardo vas não querer obedeser lhe mãdara notefiquar segunda ves que com Pena de sinquoenta cruzados se fizese Prestes pera por todo este mes de setembro fazer a dita viagem com pena de sincoenta cruzados $\mathrm{E}$ ora $\mathrm{O} \mathrm{Ca}-$ pitam e guovernador Rui vas pinto the tinha mandado notefiquar que loguo dezestise das dittas noteficasois $\mathbf{E}$ allevantase a dita pena ao dito mestre dizendo que a elle se avia de Pedir a dita embarcasão $\mathbf{E}$ a elle pertençia ttomada pera o dito efeito tomandolhe a elle dito Provedor $\mathrm{E}$ metendose em sua jurdissam afin dese emcontrar con 12r..$^{\circ}$ elle e de // ho molestar $\mathrm{E}$ impedindo por todas as vias que náo sirva seu oficio $E$ uze da dita jurdisam pois o dito admenistrador the tinha feito a dita Petisão $\mathbf{E}$ a elle pertensia dar lhe Provimento $E$ mandar fazer nno cazo as despezas que sua magestade lhe concede per sua Provizão de que tudo mandou a min escrivam lhe dese E lansase aqui minha fee com a do escrivão do almoxarifado ssebastião coelho dami $\mathrm{E}$ do meirinho do mar fran.co da Costa com a mais prova que nesesaria for Pera sua guoarda fran.co da Costa escrivão da provedoria da fazenda o escrevj - Dioguo lopes de bulham -

$\mathrm{E}$ sendo aos vinte e sinquo dias do mes $\mathrm{E}$ anno atras declarado pello caPitam e guovernador $R u j$ vaz pinto foi mandado a min escrivão e ao escrivão do almox.do sebastião Coelho notteficasemos ao provedor da fazenda Dioguo lopes de bulham que atee oie ao meio dia mandaçe allevantar a lionardo vas a pena $\mathbf{E}$ notefiquasão que the tinha mandado fazer ssobre a em // barcasão que the tinha mandado dise pera o admenistrador hir a sam Visente sob pena que não o fazendo asi o avia por encorri$12 v^{\circ}$ do em pena de dous mezes de prizão em hua fortaleza de sincoenta cruzados pera forteficasão della a quoal noteficasão eu escrivão dou por fee fazer ao dito Provedor oje as nove oras antes do jantar E loguo Pello dito Provedor em prezensa de min escrivão foi mandado ao meirinho do mar fr.co da costa que loguo de sua parte fose allevantar ao dito lionardo vaz as noteficassois que the tinha mandado faser dizendo que elle o fazia por Remir sua avechasão $\mathrm{E}$ por se não emcontrar com o dito $\mathrm{Ca}$ pitan mor por quoanto seu jntento era fazer lhe moles- 
tias E não Por intender que o cazo pertencia ao ditto $\mathrm{Ca}$ pitan mor se não a elle dito Provedor por o ter ameasado por muitas vezes de que pedia a min escrivão lhe pasase as certidois $E$ fees que nesesarias the fosen $\mathbf{E}$ declaro que o dito capitan mor aos vinte $\mathbf{E}$ quoatro dias do mes

13r.0 de setembro a tarde mandou que a dita noteficasão se fizese oie pella // manhãn que forão vinte $E$ sinquo fran.co da Costa escrivão da provedoria da fazenda a escrevj fr. co da Costa barros -

Sertefiquo eu sebastião coelho da min escrivão do almoxarifado $\mathbf{E}$ alfandegua por sua magestade que o Cobteudo asima $\mathrm{E}$ atras dou por fee he Paso na verdade $\mathrm{S}$ asin o dou Por fee Rio de janeiro vinte e sinquo de setembro de seissentos e dezanove annos - Sebastião coelho damin - o qual treslado de autos certidões e t.as eu Jorge de Sousa t.am Publiquo Do judisial e notas nesta sidade de São Sebastião $R j$ de jan.ro Por sua mag.de fis tresladar Dos ProPios a que me RePorto bem E fielm.te $\mathrm{E}$ a outra via que tenho Pasado Per com elle ocorrer $\mathbf{E}$ consertar e con o ofisial aqui asinado e dizem as duas entrelinhas "do" chamar a sua caza $\mathbf{E}$ sendo la me mandou e os mal escritos dizem "deste Pre" se fes e pera o que fis Por verdade oje vintahu de dezembro de mil e seissentos e dezanove annos Jorge de Sousa.

Consertado Per $\mathrm{mj}$

t.am Publiquo

Jorge de Sousa

Comigo escrivão da ouvidoria

D.o Da fn.ca. 